\title{
Échelles diffuses: Prospectives de conception via matière et VR
}

\author{
Églantine Bigot-Doll ${ }^{1}$ \\ MAP-Aria, ENSA de Lyon, France
}

\begin{abstract}
Résumé. Dans le cadre d'un enseignement de projet en école d'architecture, nous investiguons ici la question de l'échelle entre monde physique et environnement virtuel. Les objectifs de conception prospective de l'enseignement en question ont permis d'observer les relations entre immersions analogiques et digitales sous le prisme de l'échelle tantôt perdue, tantôt redéfinie dans une quête de matérialité spéculative.
\end{abstract}

Mots-clés. Conception, Numérique, Prospective, Échelle, Perception.

\begin{abstract}
The didactic situation of a design studio in a school of architecture allows for the investigation of scales between physical world and virtual environments. The prospective conception of this studio aimed at observing the relations that exist from analog to digital immersions studied through the prism of a scale that is sometimes lost and sometimes redefined, in search of a speculative materiality.
\end{abstract}

Keywords. Conception, Digital, Prospects, Scale, Perception.

\section{Introduction}

La présente recherche, poursuivant des travaux de thèse de doctorat en architecture et ancrée dans un cadre pédagogique, porte sur la conception architecturale que nous qualifierons ici de prospective. Celle-ci tente d'observer le développement de créativité par hybridations successives d'outils numériques et analogiques au moment de la conception. Les différentes échelles des représentations successives sont biaisées par la dimension $a$ scalaire induite par le numérique. Ainsi, en vue d'interroger des usages et matérialités architecturaux émergents, nous tenterons de mettre à profit les confusions impliquées par ces échelles dorénavant diffuses, notamment via le dispositif de réalité virtuelle confronté à la confection manuelle.

\footnotetext{
${ }^{1}$ Eglantine Bigot-Doll : eglantine.bigot-doll@lyon.archi.fr
} 


\section{Rétentions matérielles}

\subsection{Miniatures}

Nous empruntons ici le concept des rétentions ${ }^{2}$ de Bernard Stiegler pour caractériser les productions transitionnelles nécessaires à la conception. Qu'elles soient de nature physique ou issues de la CAO, voire même de la CFAO (virtuelles puis physiques), celles-ci sont imprégnées d'un signifié architectural à chaque fois remodelé, altéré, soumis aux mutations de l'outil tout au long de l'élaboration du projet.

Ainsi, les rétentions tertiaires en tant que «supports de mémoire extérieure à la psyché », sont un premier attendu pédagogique crucial en termes de stratégie immersive. Chaque groupe d'étudiants se voit attribuer un nuancier établi à partir des thématiques d'investigation évoquées après lecture d'une nouvelle de Borges ${ }^{3}$. Compte tenu de ce nuancier, l'objectif est d'introduire la notion de déclinaison par confection de miniatures dont l'échelle serait celle de la pilule.

Nous illustrerons les résultats issus des séquences pédagogiques à travers la production représentative d'un groupe d'étudiants de première année de master en architecture dans le cadre d'un enseignement de projet ${ }^{4}$.
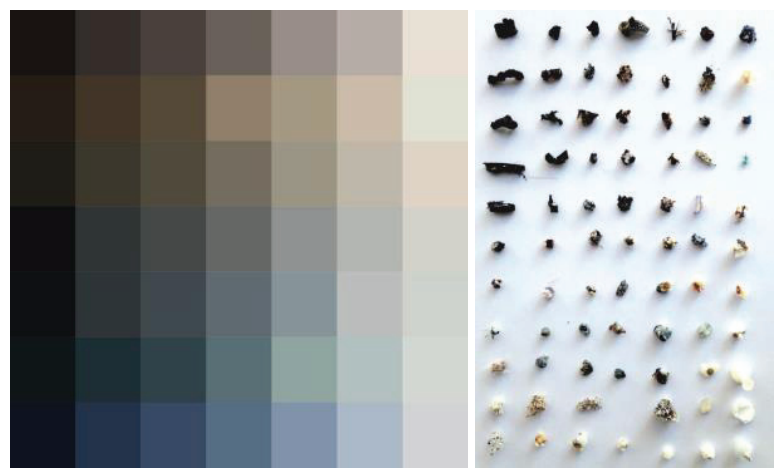

Figure 1. Nuancier imposé / Déclinaisons rétentionnelles / Individu prélevé.

Dans la figure 1, le nuancier «bleu et ivoire » imposé donne naissance à une série d'objets «pilules ». La troisième image montre une pilule extraite de ce nuancier. Chaque objet issu de cette première série, en tant que projectile $n^{0}$ est rétention de l'intention primitive des concepteurs. À ce stade, les contraintes uniques initiant l'exercice sont :

- l'identification d'une thématique à investiguer suite à la lecture du texte de Borges : ce groupe d'étudiants choisit la thématique du miroir et de l'illusion dans ce premier temps.

- la série de projectiles $n 0$ dite « pilules ». Elle doit être fidèle au nuancier imposé.

\footnotetext{
2 «Les rétentions sont ce qui est retenu ou recueilli par la conscience. Ce terme est emprunté à Husserl ; mais les rétentions tertiaires sont propres à la philosophie de Bernard Stiegler. Les rétentions sont des sélections [...] »-Ars Industrialis - association internationale pour une politique industrielle des technologies de l'esprit; extrait de la page http://arsindustrialis.org/r\%C3\%A9tention

${ }^{3}$ Borges, J. L. (2007). L'attente. In L'aleph (pp. 173-179). Paris : Gallimard.

${ }^{4}$ Étudiants du groupe REFLECT : Camille Berube ; Marta Fazzino \& Robin Michel - semestre d'automne 2017-2018.
} 


\subsection{Atmosphères et empathie}

Les miniatures produites en tant que rétentions tertiaires, fragments de mémoire extérieure aux concepteurs d'une part, influencées par le nuancier imposé d'autre part, portent plusieurs objectifs. Elles permettent d'introduire la notion de paramétrique par une stratégie analogique, évacuant ainsi l'appréhension envers l'outil numérique. Les paramètres sont : [outil ; geste ; matière] dans un territoire colorimétrique donné et selon la contrainte d'échelle.

Le second objectif, non annoncé explicitement aux étudiants, est la propriété déterritorialisante de la miniature en confection. Ici le voyage ne s'opère pas par la distance mais par l'échelle des replis de la matière pendant le faire (Bigot-Doll, 2018). Ce dispositif rétentionnel est une immersion dans des matérialités générées par individuation technique d'une part, émergence d'univers insoupçonnés, et ainsi, d'usages singuliers d'autre part. Chaque objet miniature encapsule des univers vecteurs de percepts et dans lesquels les étudiants projettent des sensations, des chimères, des fictions (figure 3), des réalités spéculatives. La caractéristique essentielle, identifiée comme vectrice de déterritorialisation dans cette expérience, réside dans une forme de fascination envers une esthétique singulière que l'on pourrait qualifier d'empathie. L'empathie esthétique (Lemarquis, 2014) d'une part, étayée par l'empathie via le toucher (Braun, Fontanella, et Stringari, 2015) se combinent dans cette immersion par l'échelle micro.

En somme, le territoire intérieur, le paysage spéculé d'abord individuellement puis au sein du groupe est porté par ces objets. Objets sensuels et réels à la fois, la série initie les tensions nécessaires à l'émergence de leurs qualités eidétiques (Harman, 2010), elles participent à la mise en place d'une atmosphère particulière de projet.

\subsection{Condenser pour décupler}

Ainsi la miniature, par essence, comprime les percepts. La déclinaison imposée par la dimension paramétrique et le nuancier imposé obligent l'étudiant à répéter des gestes, investiguer des phénomènes décuplés dans chaque objet. Puis l'intention, la conation, mutent sous l'effet de la verbalisation, la représentation par les mots exigée à chaque étape de la conception sous forme de fiche.

Les étudiants dépassent alors les percepts premiers en échafaudant intérieurement des univers et des récits en tant que fictions d'un imaginaire comprimé et pourtant intensifié. L'objet inclue et se dilate, à l'image du moment quelconque de la fiction littéraire dont Jacques Rancière (Rancière, 2017) fait état dans son ouvrage récent :

“ [...] le moment qui ne construit ni ne détruit plus rien, qui ne se tend vers aucune fin mais se dilate à l'infini, incluant virtuellement tout temps et tout lieu autre. Un temps de la coexistence, gagné par la libéralité de l'espace " (Rancière, 2017, p. 131).

Cette immersion compressive est intensifiée par les itérations dans la séquence, on pourrait ainsi la qualifier d'immersion compressive-itérative. À l'image des séries d'expansions qui ont succédé aux séries de compressions de la matière du sculpteur César, nous choisissons d'aborder l'environnement numérique par un dispositif de réalité virtuelle (VR). Celui-ci perturbera le rapport à l'objet par abolition de sa matérialité d'une part, et, d'autre part, par l'échelle à présent déterminée par l'étudiant. 


\section{Distensions digitales}

\subsection{Le virtuel a-scalaire}

Si la confection de miniatures rétentionnelles opère une immersion compressive et répétitive par condensation de matière, la perte de l'échelle originelle impliquée par le dispositif de réalité virtuelle opère quant à elle une forme de distension de l'espace. On observe que l'objet $n^{0}$ scanné (désormais $n^{l}$ ), à présent dépourvu de sa matérialité, est systématiquement agrandi par les étudiants au sein de l'environnement virtuel de manière à atteindre une hauteur au moins égale à la taille de l'utilisateur. Le modèle est dorénavant « englobant» et opère à son tour une forme d'immersion que nous qualifierons d'expansive (figure 2).

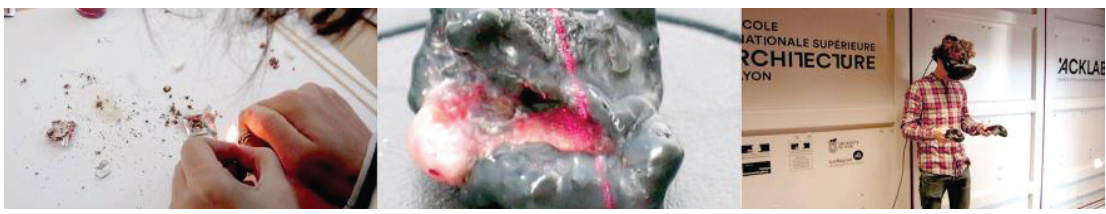

Figure 2. Confection / Scan 3d / Import VR.

\subsection{Mal de VR?}

Physiologiquement, il arrive que la réalité virtuelle provoque une cinétose qui, dans le cas de l'utilisation d'un oculus, pourrait être définie comme un mal de mer "inversé »: le cerveau reçoit une information qui ne correspond pas à ce que le corps perçoit. Les décalages perceptifs impliqués par ce dispositif - bien que n'atteignant que rarement l'intensité du motion sickness avéré - perturbent en effet les sens, et l'aptitude retentionnelle de la psyché se voit ainsi amplifiée. Les distensions-distanciations scalaires successsives modifient le rapport à l'objet initialement confectionné par la main et désinhibent les potentialités dans la conception par émergence de prospects de matérialité et d'usages ${ }^{5}$ via la géométrie virtuellement «manipulée » (figure 5).

Toutefois, il a été évoqué à quelques reprises au cours de la séquence, une forme d'inconfort voire de malaise au moment de côtoyer l'objet autrefois minuscule et délicat, désormais exporté dans l'environnement virtuel aseptisé.

\subsection{Promiscuité a-haptique}

Si la promiscuité virtuelle prive toutefois l'utilisateur du contact avec l'objet, une empathie certaine se crée par jeux d'échelles selon un référentiel d'échelle anthropologique (Kapp, 2007) en tant que rapport à un soi physiométrique (Bigot-Doll, 2017). C'est-à-dire que, paradoxalement, l'échelle transformée de l'objet permet à l'étudiant de l'appréhender dans une forme de réalité nouvelle, accrue du fait de ses mensurations désormais comparable à la taille humaine.

Ainsi les modifications d'échelles réalisables uniquement au sein de l'espace virtuel et $a$ posteriori permettent dès lors de circonscrire un champ des possibles dont les dimensions sont établies - consciemment ou non - par un rapport au corps du concepteur. De plus, la

\footnotetext{
${ }^{5}$ Les usages prospectifs qui émergeront plus tard dans l'enseignement sont axés au sein des différents groupes, sur l'exploration alternative des lieux de sommeil diurne, de grottes urbaines, de pédagogie générative.
} 
quasi-totalité des étudiants, au cours de l'expérience de VR, verbalise la sensation de pouvoir «toucher» le modèle $3 \mathrm{~d}$. Malgré l'absence de contact haptique, chaque individu projette une promiscuité avec l'objet contemplé, parcouru et palpé par la pensée.

Tout au long du développement de projet qui suivra, le rapport au modèle $3 \mathrm{D}$ - visible uniquement à l'écran en temps «normal»-se trouve singulièrement modifié au profit d'une conation certaine envers l'expérience potentielle d'un espace autre. Dans le cas du groupe d'étudiants dont la production illustre le présent article, le regard porté sur la thématique évolue depuis l'analogie du miroir initiale vers une exploration architecturale de la perception par le toucher, éludant alors la suprématie des sensations rétiniennes. Le reflet porte à présent une dimension haptique, concrète, palpable.

Le modèle - paramétrique, complexe, à courbure variable, etc. - qui autrefois impliquait plutôt inhibition et appréhension car davantage assimilé à un besoin de précision, de mensuration et de représentation, est, depuis l'expérience de VR qui a initié le semestre, vecteur de projection de matérialités alternatives, émergences de sensations par conception de bestiaires de typologies architecturales (figures 3 et 4).
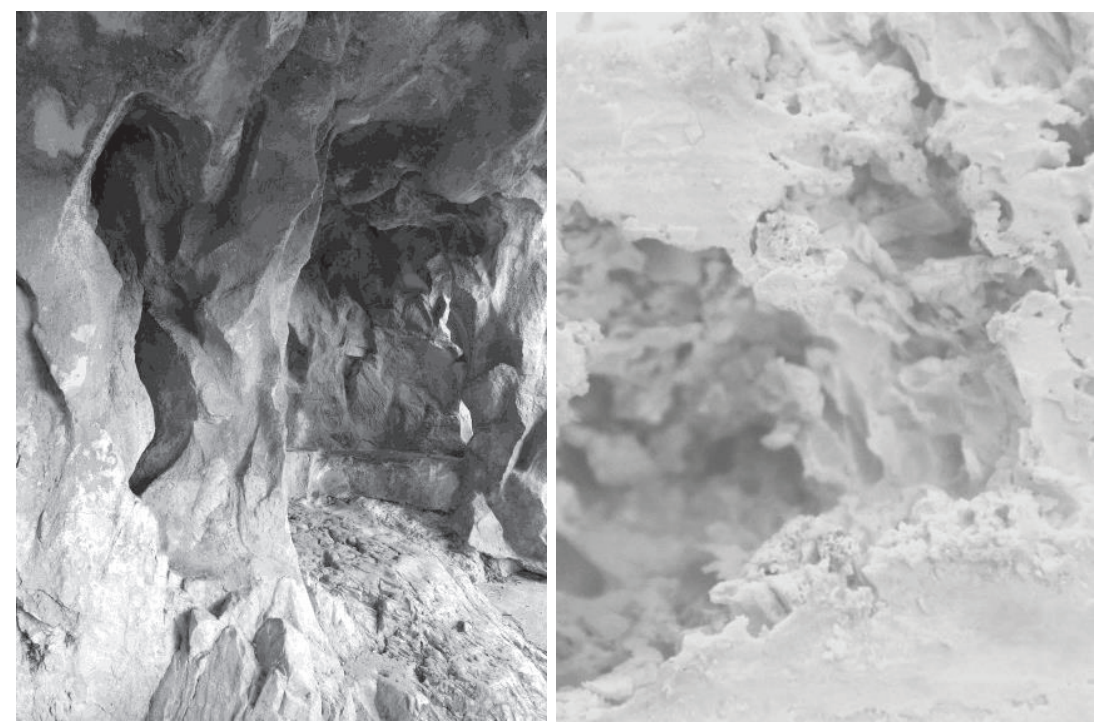

Figure 3. Univers macro-micro.

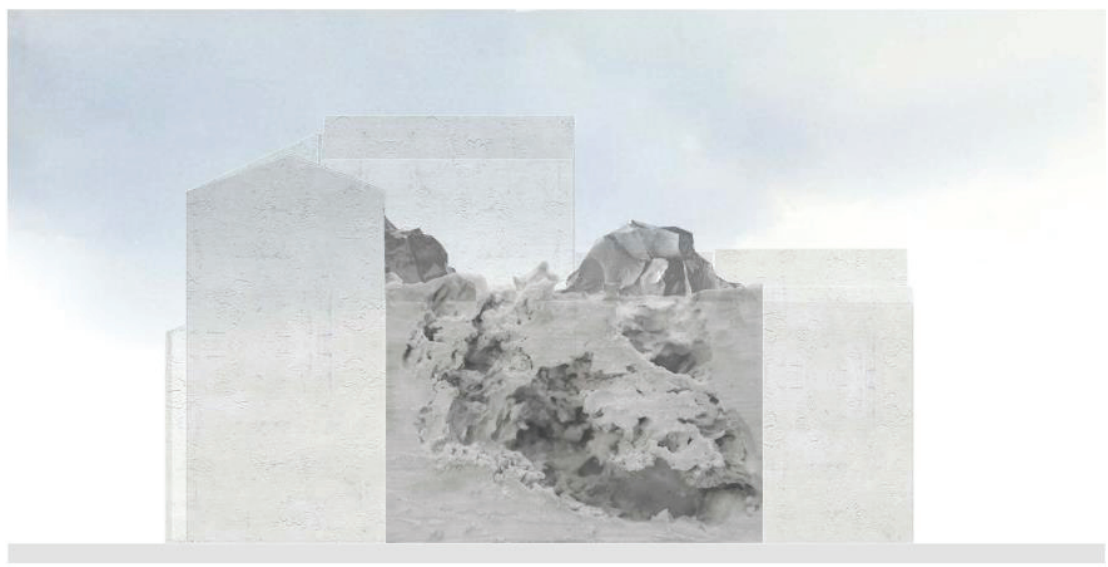

Figure 4. Coupe d'intention. 


\section{Cycles antalgiques}

L'(ap)préhension d'un environnement autre via les modèles virtuels et spécifiquement par la VR, constitue selon les neurosciences une réduction de l'inhibition dans une situation normée - celle de l'enseignement de projet architectural par exemple - voire une action antalgique. Cette action antalgique est d'une telle intensité que la chirurgie, grâce entre autre à la VR, peut aujourd'hui s'affranchir d'une anesthésie générale lors d'interventions lourdes à cerveau ouvert (Martin, 2016).

En effet, les ressources attentionnelles nécessaires à l'immersion dans un environnement virtuel semblent mobiliser perception, et dans notre cas, émotions en décuplant leurs effets. La représentation dans l'espace immersif interne ou externe, dans la psyché comme dans un environnement virtuel, n'aurait plus seulement vocation à aider une modélisation dans la conception, mais par succession d'expériences immersives tantôt compressives tantôt expansives, de favoriser l'émergence de prospects par désinhibition scalaire.

Nous avons choisi d'illustrer cet article à travers l'exemple de la production d'un groupe unique. Toutefois, au sein de cette recherche qui vise à encourager l'émergence de postures prospectives dans le projet d'architecture, nous avons observé que l'ensemble des productions interroge l'espace de la perception, en particulier la perception non rétinienne. Le sens du toucher occupe une place centrale dans la tonalité des projets : le toucher pour faire, l'espace enveloppant et soyeux d'un lieu de sommeil, le toucher rêche du minéral, humide et vaporeux des bains. Ainsi l'effet antalgique causé par les situations immersives successives impliquerait-il une amplification des qualités haptiques et soma-perceptives de matérialité architecturale conçue?
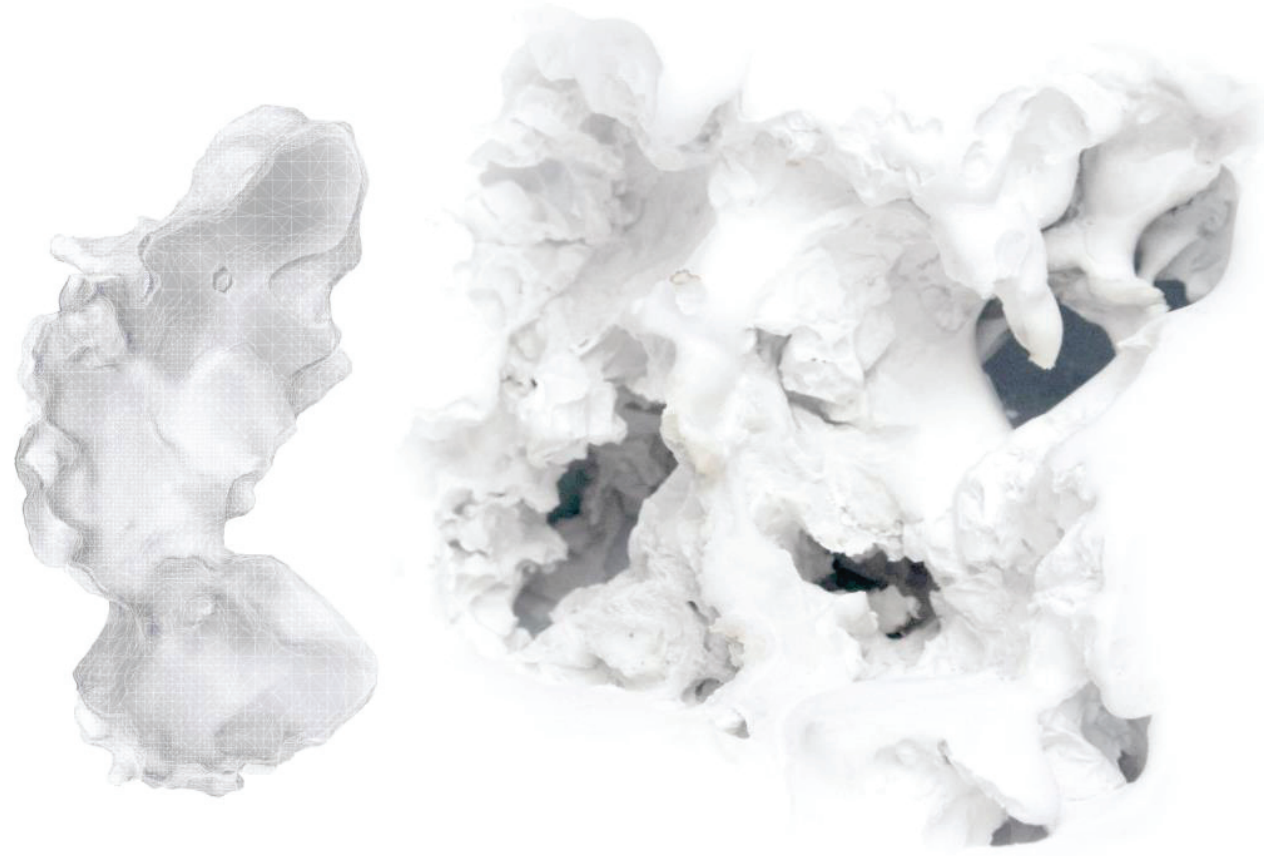

Figure 5. Modèle virtuel du vide par scan d'un objet en cire / Maquette obtenue par moulage de l'objet en cire immergé puis fondu. 


\section{Conclusion}

Du monde physique et micro aux environnements virtuels, qu'ils soient écrans ou oculus, la conception de projet à l'échelle architecturale immerge le concepteur dans des univers et interfaces fortement hétérogènes. Les itérations entre immersions compressives et expansives impliquent une redéfinition permanente de la forme en devenir et, dans une dimension prospective, mêlent des réalités amendées selon les termes de Ferda Kolatan ${ }^{6}$ (Young, 2015). La verbalisation - par analogies, chimères voire fictions - incarne des postures architecturales émergentes de ces spéculations rétentionnelles. De proche en proche et par contagion (Foucault, 1966), les similitudes entre matière singulière et images familières nourrissent la conception au sein d'un cycle expérientiel dont les paradigmes ont muté sous l'action du numérique. Aujourd'hui les dispositifs tels que la réalité virtuelle mais aussi la robotique nous offrent la possibilité d'un renouvellement de la pédagogie dans les écoles d'architecture davantage axé sur une compréhension par le corps.

Remerciements aux étudiants de MA[A]D, à l'équipe NOVA, Elsa Trincal, Laura Michal, Cécile Perriche, Fabrice Cavaillé, Christophe Gonnet, Marian Janda, Kevin Jacquot, Anna-Andréa ObéGervais.

\section{Bibliographie}

Bigot-Doll, E. (2017). Du Noumène à l'Ekkoumène, une émulsion analogique-numérique pour une prospective du projet d'architecture. Université Lumière Lyon II, ENSA de Lyon.

Bigot-Doll, E. (2018). Du figment au fragment, Miniature et conception architecturale. In I. Roussel-Gillet \& E. Thoizet (Éd.). Présenté à Colloque international : La miniature, un dispositif artistique et un modèle épistémologique à l'ère du nano, Maison de la recherche - Université d'Artois, Arras : Brill.

Braun, E. et al., (2015). Touch and Empathy. In Alberto Burri : The Trauma of Painting (p. 50-59). New York, NY: Solomon R. Guggenheim Museum \& Kunstsammlung Nordrhein-Westfalen (Germany).

Foucault, M. (1966). Chapitre 2 : La prose du monde - Les quatre similitudes. In Les mots et les choses; une archéologie des sciences humaines. (pp. 32-40). Paris : Gallimard.

Harman, G. (2010). L'objet quadruple une métaphysique des choses après Heidegger. (O. Dubouclez, Trad.). Paris : Presses universitaires de France.

Kapp, E. (2007). Principes d'une philosophie de la technique. (G. Chamayou, Trad.). Paris : Librairie philosophique J. Vrin.

Lemarquis, P. (2014). L'empathie esthétique, la pensée analogique est une pensée magique. In Portrait du cerveau en artiste (p. 212-237). Paris : Odile Jacob.

Martin, N. (2016, octobre 19). Réalité virtuelle: une révolution scientifique [Podcast]. La méthode scientifique. France Culture. Consulté à l'adresse :

https://www.franceculture.fr/player/export-reecouter?content=7dfde045-8a14-41e2$96 \mathrm{~d} 1-855 \mathrm{~d} 4 \mathrm{e} 1617 \mathrm{e} 1$

Rancière, J. (2017). Les bords de la fiction. Paris: Éditions du Seuil.

Young, M. (2015). The estranged object (Graham Foundation for Advanced Studies in the Fine Arts). Young et Ayata.

${ }^{6}$ En anglais amended realities, selon l'expression originale. 